Research Article

\title{
Single-Photon Emission Computed Tomography Image-Assisted Diagnosis of Thyroid Diseases under Convolutional Network Neural Algorithm
}

\author{
Shaobo Chen $\mathbb{D}^{1},{ }^{1}$ Yinzhen Pi $\mathbb{D},{ }^{1}$ Haiyan Gong $\mathbb{D}^{1},{ }^{1}$ Huaijun Wang $\mathbb{D}^{1},{ }^{1}$ and Shu Liu $\mathbb{D}^{2}$ \\ ${ }^{1}$ Department of Endocrinology and Metabolism, Affiliated Changsha Hospital, University of South China, Changsha 410005, \\ Hunan, China \\ ${ }^{2}$ Department of Endocrinology and Metabolism, The First Affiliated Hospital of Xi'an Jiaotong University, Xi'an 710000, \\ Shaanxi, China
}

Correspondence should be addressed to Shu Liu; 141003330237@st.sdju.edu.cn

Received 30 September 2021; Revised 12 November 2021; Accepted 15 November 2021; Published 16 December 2021

Academic Editor: M Pallikonda Rajasekaran

Copyright (C) 2021 Shaobo Chen et al. This is an open access article distributed under the Creative Commons Attribution License, which permits unrestricted use, distribution, and reproduction in any medium, provided the original work is properly cited.

\begin{abstract}
The aim of this study was to investigate the value of single-photon emission computed tomography (SPECT) based on the convolutional neural network (CNN) algorithm in thyroid diseases. Thirty-five patients with thyroid disease from the hospital were selected as the observation group, and another 35 healthy volunteers were selected as the control group. The constructed model of SPECT based on the CNN algorithm was compared with the backpropagation neural network (BPNN) algorithm, which was then applied to the SPECT of 35 patients with thyroid disease. It turned out that as the number of iterations increased, the parameter training of CNN was gradually sufficient, the network model was continuously optimized, and the accuracy gradually increased. From the data results, the Dice value of the proposed CNN algorithm was higher than that of the BPNN algorithm and the segmentation effect was relatively good. The visual index of the thyroid/neck of the observation group $(2.68 \pm 1.32)$ was remarkably inferior to that of the control group (12.347.54) $(P<0.05)$. The visual index of the thyroid/submandibular gland in the observation group $(1.02 \pm 0.41)$ was remarkably inferior to that of the control group $(8.89 \pm 4.86)(P<0.05)$. The visual index of the thyroid/parotid gland in the observation group $(1.04 \pm 0.58)$ was remarkably inferior to that of the control group $(8.53 \pm 4.25)$

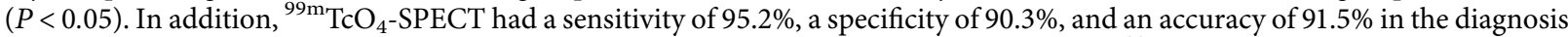
of thyroid diseases. The area under the curve of the receiver operating characteristic curve for ${ }^{99} \mathrm{mcO}_{4}$-SPECT diagnosis of thyroid disease is 0.958 , and the $95 \%$ confidence interval is $0.834 \sim 1$. In summary, the SPECT based on the CNN algorithm proposed in this study has a good segmentation effect and can accurately locate the anatomical information of thyroid diseases, which can replace the traditional diagnostic methods for the diagnosis of thyroid diseases.
\end{abstract}

\section{Introduction}

The thyroid gland is a very important gland in the human body. It is located on both sides of the neck and can secrete thyroid hormones [1]. Thyroid hormones play a vital role in the body. Both endocrine and metabolism of the body require the balance of thyroid hormones. Therefore, thyroid diseases will have a greater impact on the human body. Common thyroid diseases include goiter, hyperthyroidism, hypothyroidism, thyroiditis, thyroid nodules, and thyroid cancer [2]. In the past, most thyroid examinations were carried out by palpation. Due to the position and size of the nodules in the thyroid, the patient's neck length, obesity, and the examiner's experience, the probability of thyroid disease was not high. With the increase in the frequency of inspections and the increase in the sensitivity of inspection methods, the prevalence of thyroid diseases has increased year by year [3].

Clinically, ultrasound is widely used in the diagnosis of thyroid diseases, with the advantages of nonseminal, simple operation, and strong reproducibility [4]. CT scanning is difficult to distinguish between hypothyroidism, thyroid 
cancer, and thyroid tumors. If clinicians do not have sufficient experience, they are likely to miss the diagnosis and misdiagnose [5]. The above examination methods mainly observe the anatomical changes of the lesion, while nuclear medicine combines function and anatomy to accurately locate, stage, and prognosis [6]. In addition, single-photon emission computed tomography (SPECT) is the most widely utilized nuclear medicine examination in clinical practice [7]. SPECT can accurately locate the location of the lesion and present the physiological, biochemical, and organizational information of the lesion in the form of images in a systematic and comprehensive way. With the continuous development of imaging technology, the diagnostic efficiency of SPECT imaging for benign and malignant thyroid lesions is gradually improved. Among them, ${ }^{99} \mathrm{mcO}_{4^{-}}$ SPECT imaging can directly reflect the anatomical and functional information of the thyroid gland, with simple operation and strong reproducibility, which is of great significance for the early diagnosis and prognosis of thyroid diseases [8].

Deep learning is a learning method close to artificial intelligence in machine learning, in which a convolutional network neural algorithm $(\mathrm{CNN})$ is a deep discrimination model. CNN algorithm is a truly successful learning algorithm for training multilayer network structure, which can satisfy the preprocessing of minimized data and carry out implicit extraction of features in the hidden layer of the deep network [9]. Image processing is based on deep learning $\mathrm{CNN}$ algorithm, which not only greatly improves the accuracy of image recognition, but also avoids the need to consume much time for manual feature extraction so that the efficiency of online operation is greatly improved.

In this study, the CNN algorithm was compared with the backpropagation neural network (BPNN) and applied to SPECT images of 35 patients with thyroid disease to evaluate the diagnostic value of ${ }^{99} \mathrm{mcO}_{4}$-SPECT in thyroid diseases and provide data support for the diagnosis of early thyroid disease by SPECT based on CNN algorithm.

\section{Materials and Methods}

2.1. Subjects. In this study, 35 patients with thyroid disease hospitalized from January 2019 to December 2020 were selected as the observation group and diagnosed by SPECT based on the CNN algorithm; another 35 patients with thyroid disease were selected as the control group and diagnosed by conventional CT during the same period. The obtained CT images were compared with the existing normal thyroid CT images in the hospital. There were 31 males and 39 females, with an average age of $38.62 \pm 12.34$ years. This study had been approved by the Ethics Committee of the hospital, and the included subjects or their family members had signed the informed consent.

Inclusion criteria are as follows: (i) patients who had not received treatment before medical treatment and did not take drugs that interfered with the experiment; (ii) patients with complete clinical data of thyroid function examination; (iii) patients aged between 20 and 65 years.
Exclusion criteria are as follows: (i) patients with complicated mental illness; (ii) patients with abnormal liver and kidney function; (iii) patients who did not cooperate or quitted midway; (iv) patients with hypertension, hyperglycemia, and diabetes; (v) patients without follow-up and with incomplete clinical data.

\subsection{Observation Indexes and Diagnostic Criteria.} Outcome measures are as follows: general information of patients with thyroid disease was recorded, including gender, age, and classification of thyroid disease; diagnostic criteria are as follows: thyroiditis is characterized by thyroid goiter and thyroid peroxidase antibody (TPOAb) or thyroglobulin antibody (TgAb); and hyperthyroidism is characterized by elevated levels of serum triiodothyronine (T3) and thyroxine (T4), mild enlargement of thyroid nodules, soft glands, and asymptomatic, with enlarged glands and nodules.

The diagnosis of thyroid disease was in accordance with the 2017 American Thyroid Association guidelines for the diagnosis and management of thyroid disease [10].

2.3. ${ }^{99 m} \mathrm{TcO}_{4}$-SPECT Instrument Inspection. Patients were instructed to remove various metal objects from their body, take a supine position, raise the shoulders, and fully expose the thyroid part of the shoulder. The imaging agent was $\mathrm{Na}^{99 \mathrm{~m}} \mathrm{TcO}_{4}$. The imaging equipment was Siemens Symbia T 16 SPECT imaging instrument. Through intravenous injection of $180 \mathrm{MBq}(6 \mathrm{mCi})$ imaging agent $\mathrm{Na}^{99 m} \mathrm{TcO}_{4}$, ${ }^{99} \mathrm{~m}_{\mathrm{TcO}_{4}}$-plane imaging was performed $30 \mathrm{~min}$ after. Image scanning conditions were as follows: the low-energy highresolution collimator was employed, with an energy peak of $145 \mathrm{keV}$ and window width of $22 \%$. In addition, the matrix was $256 \times 256$, the magnification was 2.5 , and the acquisition count was $600 \mathrm{~K}$, so as to perform a SPECT tomography scan. Image extraction conditions were as follows: the lowenergy high-resolution collimator was employed, with a matrix of $256 \times 256$, magnification of $2.5,15 \mathrm{~s} /$ frame, 65 frames $/ 360^{\circ}$. CT scan parameters were voltage of $120 \mathrm{kV}$, current of $180 \mathrm{mAs}$, layer thickness of $2.5 \mathrm{~mm}$, and pitch of 0.8 . The scanning range was from the base of the skull to the entrance of the chest cavity. The built-in software of the workstation was adopted to process the SPECT extracted images, so as to obtain the same cross-sectional, sagittal, coronal, and fusion images of SPECT and CT scans.

2.4. ${ }^{99 m} \mathrm{TcO}_{4}$-SPECT Image Analysis. Three nuclear medicine physicians with rich experience in reading films independently analyzed ${ }^{99} \mathrm{TcO}_{4}$-SPECT images of the thyroid gland, observed the SPECT tomographic image and CT tomographic image, analyzed the SPECT/CT fusion image, and finally drew a conclusion. If the diagnosis opinions were inconsistent, the three physicians negotiated to make the diagnosis results consistent. After the treatment, patients with confirmed thyroid disease continued to undergo ${ }^{99} \mathrm{~m}_{\mathrm{TcO}_{4}}$-SPECT tomography. Then, the scanned images were analyzed by three nuclear medicine physicians. The 
density and recovery of the thyroid were evaluated, and the visual index of thyroid uptake (VITU) was calculated.

\subsection{Model Construction Based on CNN Algorithm.}

Figure 1 shows the CNN structure model. The structure of CNN includes the input layer, convolutional layer, downsampling layer, fully connected layer, and output layer. Some CNNs use images directly as input objects, and the size of the received image must be a fixed size. Therefore, the image size needs to be uniformly processed before the image data is input to $\mathrm{CNN}$. It is supposed that $H$ represents a grayscale image with a size of $A \times B$ containing $M$ different grayscale levels, $H_{a b}$ represents the gray value of the pixel where $H$ is a coordinate point $(a, b)$ in the image, and $\delta_{\gamma}\left(h_{a b}\right)$ represents the pixel at the position of this coordinate point, which belongs to the value of the membership degree of a certain category mark. Overall, this probability method realizes the fuzzy subset mapping from the gray image $H$ to the interval $[0,1]$, which is calculated as follows:

$$
H=\left\{h_{a b}, \delta_{H}\left(h_{a b}\right)\right\} .
$$

In equation $(1), 0 \leq \delta_{H}\left(h_{a b}\right) \leq 1, a=0,1,2, \ldots, A-1$; $b=0,1,2, \ldots, B-1$. In a grayscale image with a grayscale value of $f, g(f)$ is utilized to represent the statistical number of pixels. The relevant threshold of a gray value is determined, and the average gray level value of the background area and the target area in the gray image were calculated:

$$
\begin{gathered}
\delta_{0}=\frac{\sum_{h=0}^{s} f g(f)}{\sum_{h=0}^{s} g(f)}, \\
\delta_{1}=\frac{\sum_{f=s+1}^{s-1} f g(f)}{\sum_{f=s+1}^{s-1} g(f)} .
\end{gathered}
$$

In equation (2), $\delta_{0}$ represents the average color scale value of the background area. In equation (3), $\delta_{1}$ represents the average color scale value of the target image area, and $f$ represents the gray scale value. For the pixels with coordinate points $(a, b)$, the membership definition function is utilized, and the calculation equation is as follows:

$$
\begin{aligned}
& \delta_{H}\left(h_{a b}\right)=\frac{1}{1+\left|h_{a b}-\delta_{0}\right|+C} \quad\left(\text { if } h_{a b} \leq s\right), \\
& \delta_{H}\left(h_{a b}\right)=\frac{1}{1+\left|h_{a b}-\delta_{1}\right|+C} \quad\left(\text { if } h_{a b}>s\right) .
\end{aligned}
$$

In equations (4) and (5), the value range of $C$ is $0.5 \leq \delta_{H}\left(h_{a b}\right) \leq 1$. In the two-class segmented image, the pixels in the image belong to the background or the foreground. The $C$ value is calculated based on the maximum or minimum gray value:

$$
C=f_{\max }-f_{\min } .
$$

$\mathrm{CNN}$ is supervised learning. During network training, the output of the network is the result of manual segmentation by experts.
2.6. Evaluation of the SPECT Model. There are many indexes for determining the results of thyroid SPECT image segmentation. In this experiment, to evaluate the effect of SPECT image segmentation, the Dice coefficient was utilized as the accuracy metric. The calculation equation is as follows:

$$
\text { Dice }=\frac{2|A \cap B|}{|A|+|B|} \text {. }
$$

In equation (7), $A$ represents the gold standard of expert manual segmentation results, and $B$ represents the automatic segmentation results of the $\mathrm{CNN}$ algorithm in this research. The higher the Dice coefficient, the better the segmentation effect.

2.7. Statistical Methods. SPSS 20.0 was utilized to process the experimental data. Mean \pm standard deviation $(\square \bar{x} \pm s)$ was how measurement data were expressed. The comparison of the means between each group was performed by $t$-test. The count data were expressed as a percentage (\%). $P<0.05$ indicated that the difference was statistically considerable.

\section{Results}

3.1. Iterative Operation Process Based on CNN Algorithm. Figure 2 shows the iterative operation process of the CNN algorithm. The network solution process of CNN was realized through continuous iterative operations. If a suitable network neural model was required, it was necessary to constantly compare the experimental results of different iterations during the training process. The iteration results showed that if the number of iterations was small, the model trained by the $\mathrm{CNN}$ algorithm cannot achieve accurate classification. As the number of iterations continued to increase, the parameter training of CNN was gradually sufficient, the network model was continuously optimized, and the accuracy was gradually increased. When the number of iterations reached a certain value, the degree of change of $\mathrm{CNN}$ parameters gradually became smaller, the network convergence tended to stabilize, and the classification accuracy gradually reached the optimal value. As the number of iterations continued to increase, the training time was gradually extended.

3.2. Experimental Results Based on CNN Algorithm. Figure 3 shows the comparison of the Dice of the two segmentation algorithms. BPNN and CNN image segmentation algorithms were compared. According to the data results, the Dice value of the CNN algorithm proposed in this study was higher than that of the BPNN, and the segmentation effect was relatively better.

3.3. General Information of the Observation Group and Control Group. Figure 4 shows the comparison of general data between the observation group and the control group. The age range of the observation group was 32-49 years, and the age range of the control group was 35-49 years. There was no considerable difference in age between the two groups $(P>0.05)$. In the observation group, there were 13 


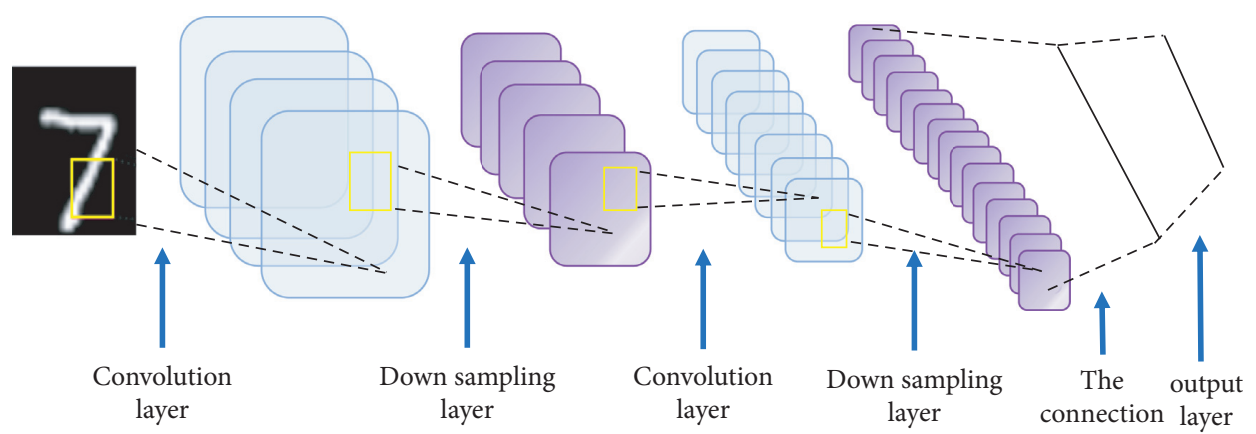

FiguRE 1: CNN structure model.

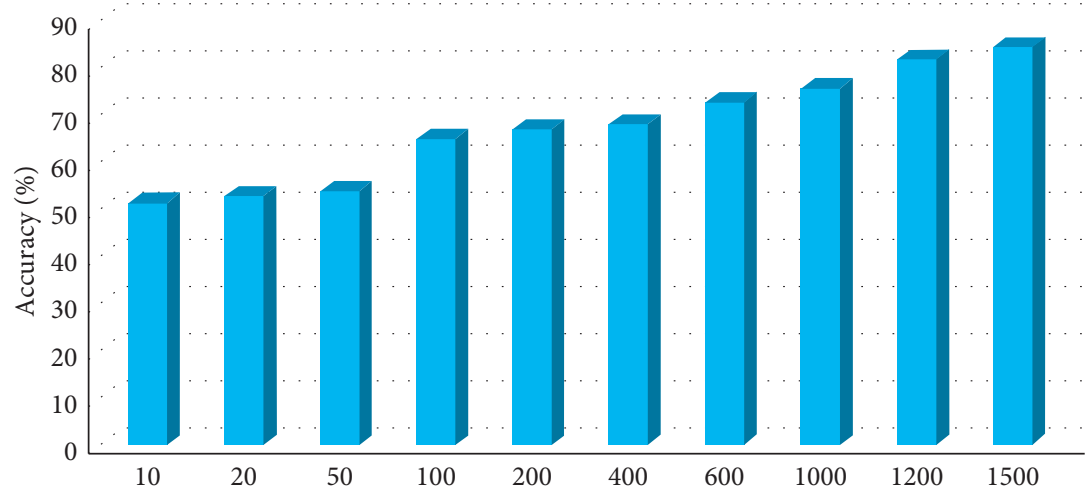

(a)

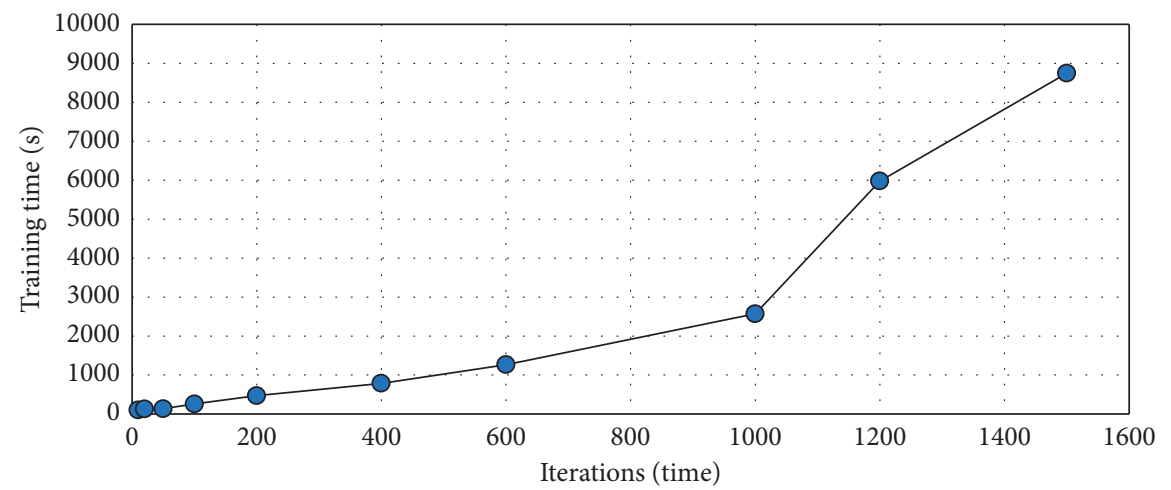

(b)

FIgURE 2: Iterative operation process of CNN algorithm. (a) Iterative accuracy; (b) iterative training time.

males (18.58\%) and 22 females (31.42\%). In the control group, there were 18 males (25.71\%) and 17 females (24.29). There was no considerable difference in gender between the two groups $(P>0.05)$. In the observation group, there were 17 cases of hyperthyroidism $(48.57 \%), 12$ cases $(34.29 \%)$ of thyroid nodules, and 6 cases (17.14\%) of subacute thyroiditis.

3.4. SPECT Images of the Observation Group and Control Group. Figure 5 shows the SPECT images of patients with normal thyroid and thyroid disease. The thyroid gland of the normal control group was clearly visible, and the position, size, and shape of the thyroid gland were normal. There was no difference in the radioactivity taken by the glands on the left and right sides, and the overall distribution was relatively uniform. The boundaries of the glands were clearly visible, and the surrounding soft tissues had relatively low radioactivity. Among them, the oral cavity and salivary glands had lower levels of radioactive ${ }^{99 \mathrm{~m}} \mathrm{Tc}$ than the thyroid. In hyperthyroidism, the entire thyroid gland was diffusely enlarged, and the function of uptake of radioactive ${ }^{99 \mathrm{~m}} \mathrm{Tc}$ was enhanced, showing uniform radioactive concentration. When there was a thyroid nodule, a $2.1 \times 1.5 \mathrm{~cm}$ low-density nodule shadow was visible in the radioactive condensed shadow of the lower extreme posterior of the thyroid. In subacute thyroiditis, the function of thyroid uptake of radioactive ${ }^{99 \mathrm{~m}} \mathrm{Tc}$ was greatly reduced, resulting in blurred images. The thyroid imaging was relatively light, and there 


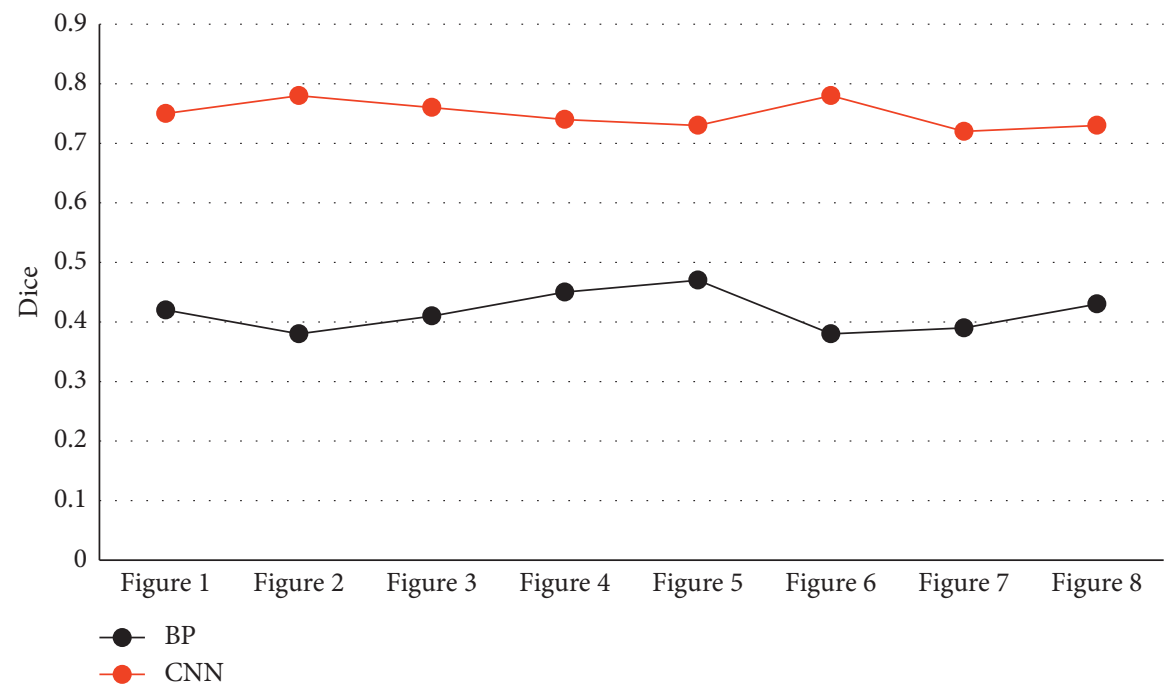

FIgURE 3: Comparison of the dice value of the two segmentation algorithms.

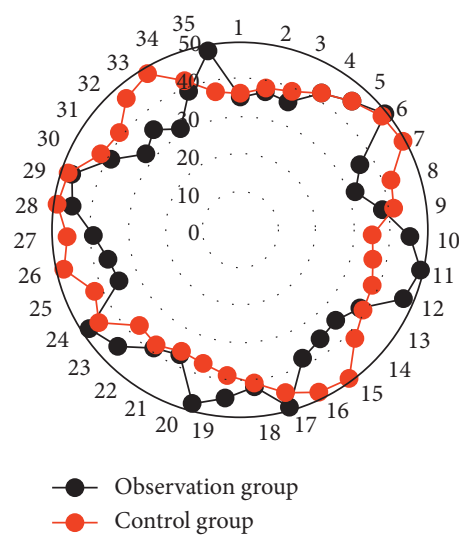

(a)



- Observation group

- Control group

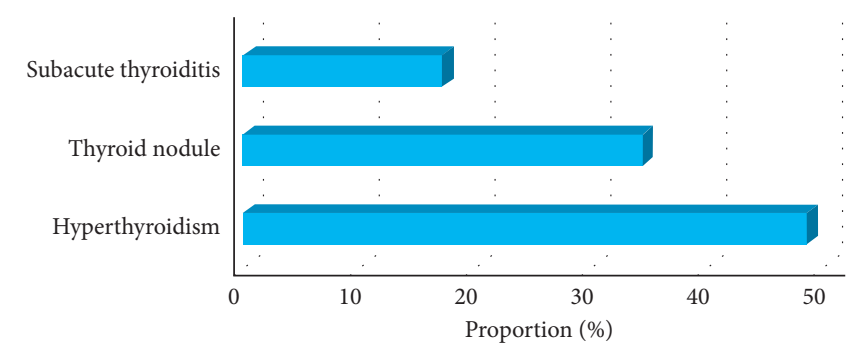

(c)

Figure 4: Comparison of general data between observation group and control group. (a) Age comparison between the observation group and control group; (b) gender comparison between observation group and control group; (c) proportion of pathological results in the observation group.

may be cases where the radioactive image distribution was not shown in the areas on both sides of the gland.

3.5. SPECT Image Result Statistics. Figure 6 shows the statistics of SPECT imaging results of patients with thyroid disease. Thyroid pathology results showed that there were 7 cases $(20 \%)$ of local lesions, of which 2 cases had no imaging $(5.71 \%)$ and 5 cases had sparse imaging $(14.29 \%)$. There were 12 cases $(34.28 \%)$ of single leaf lesions, of which 3 cases
(8.57\%) were without imaging and 9 cases (25.71\%) were sparsely imaging. There were 16 cases of double leaf lesions (45.71\%), of which 6 cases $(17.14 \%)$ were without imaging and 10 cases $(28.57 \%)$ were with sparse imaging.

3.6. Visual Index of Thyroid Uptake (VITU) Statistical Results. Figure 7 shows the comparison of VITU between the observation group and the control group. The VITU of the thyroid and soft tissue around the neck, submandibular 


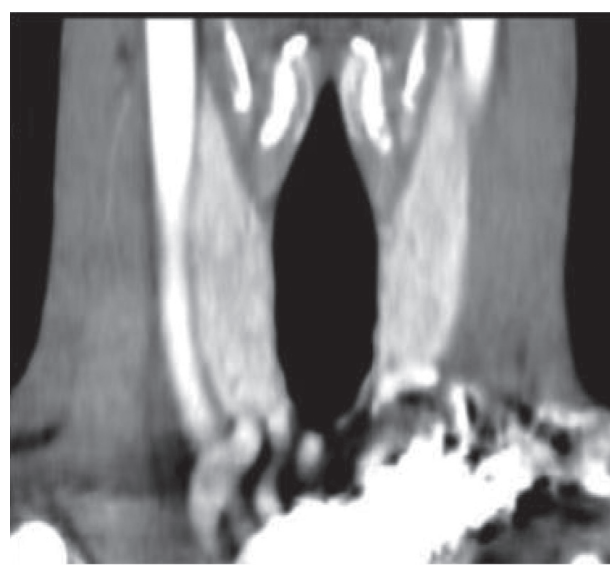

(a)

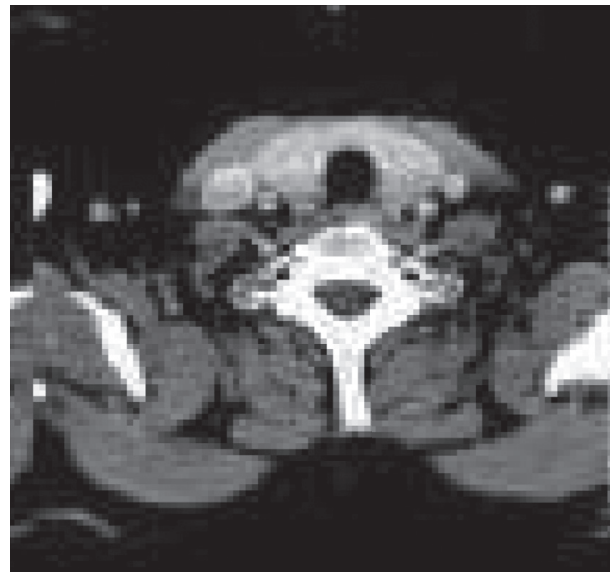

(c)

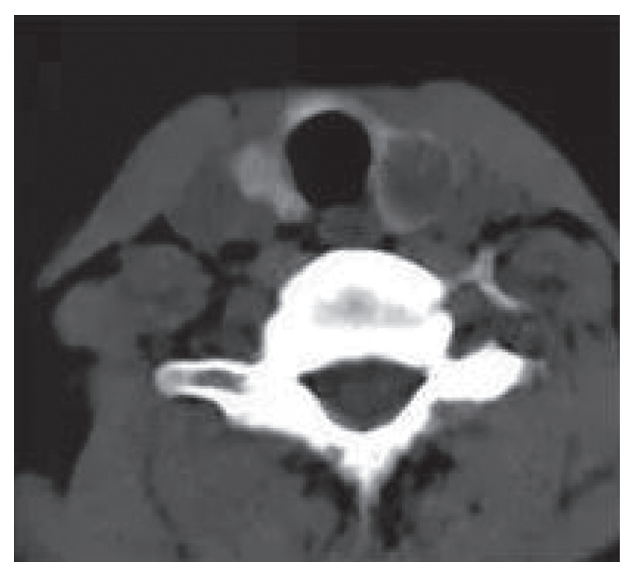

(b)

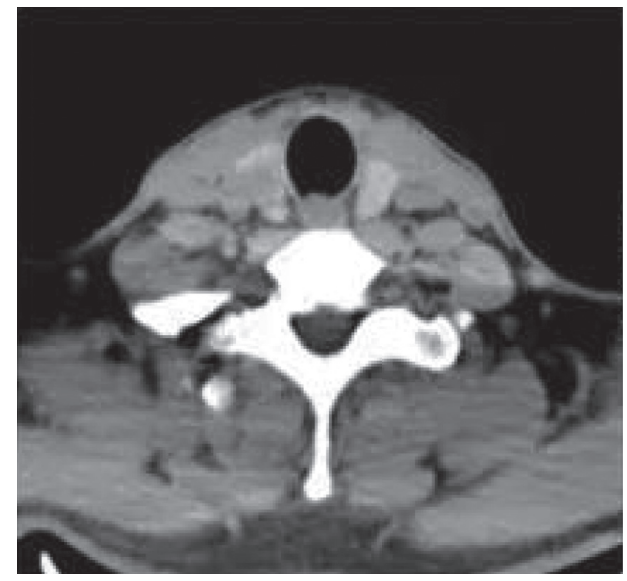

(d)

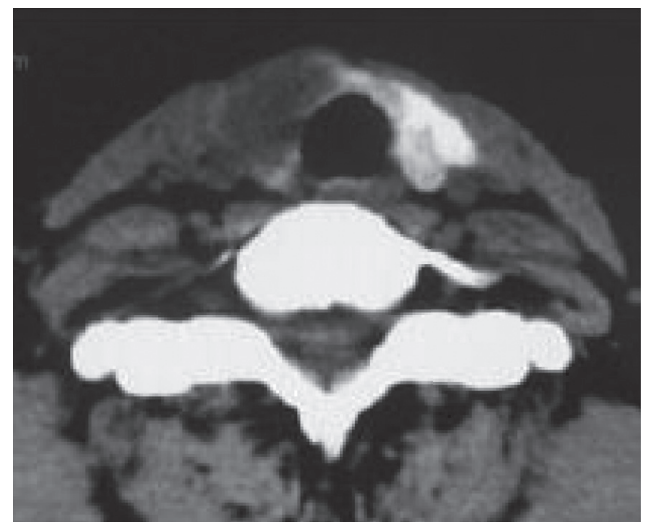

(e)

FIGURE 5: SPECT images of patients with normal thyroid and thyroid disease. (a) Normal thyroid; (b) normal lateral thyroid; (c) hyperthyroidism; (d) thyroid nodules; (e) subacute thyroiditis.

gland, and parotid gland in the observation group and the control group was calculated. The results showed that the VITU of the thyroid/neck of the observation group $(2.68 \pm 1.32)$ was remarkably inferior to that of the control group $(12.34 \pm 7.54)(P<0.05)$. The VITU of the thyroid/ submandibular gland in the observation group $(1.02 \pm 0.41)$ was remarkably inferior to that of the control group $(8.89 \pm 4.86)(P<0.05)$. The VITU of the thyroid/parotid gland in the observation group $(1.04 \pm 0.58)$ was remarkably inferior to that of the control group $(8.53 \pm 4.25)(P<0.05)$. VITU had a considerable correlation with lesion volume $(r=0.452, P<0.05)$.

3.7. Diagnostic Value of SPECT for Thyroid Diseases. Figure 8 shows the receiver operating characteristic (ROC) curve for SPECT diagnosis of thyroid disease. ${ }^{99} \mathrm{~m}_{\mathrm{TCO}_{4}}$-SPECT had a sensitivity of $95.2 \%$, a specificity of 


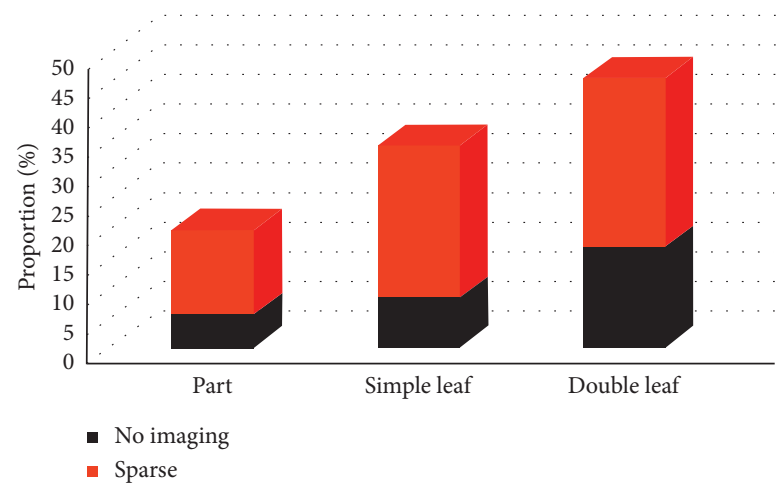

FIGURE 6: Statistics of SPECT imaging results of patients with thyroid disease.

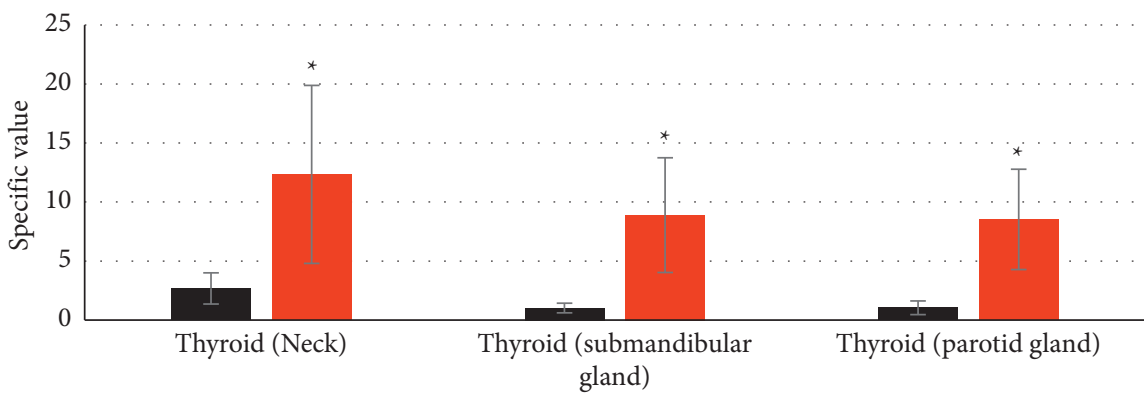

- Observation group

- Control group

(a)

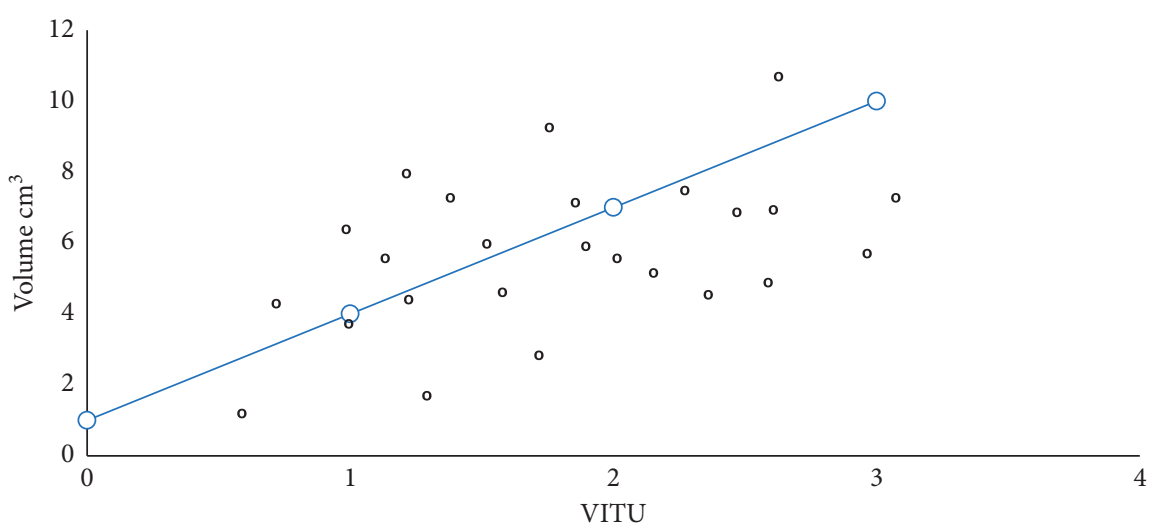

(b)

FIGURE 7: VITU statistical results. (a) VITU comparison between the observation group and the control group; (b) the relationship between VITU and lesion volume. (Note: ${ }^{*}$ indicated that compared with the observation group, the difference was substantial, $P<0.05$ ).

$90.3 \%$, an accuracy of $91.5 \%$, a positive predictive rate of $94.3 \%$, and a negative predictive rate of $89.6 \%$ in the diagnosis of thyroid diseases. The area under the curve of the ROC curve for ${ }^{99} \mathrm{mCO}_{4}$-SPECT diagnosis of thyroid disease was 0.958 , and the $95 \%$ confidence interval was $0.834 \sim 1$.

\section{Discussion}

In the early stage of thyroid disease, the severity of the disease and the scope of the lesion are inconsistent, mainly including bilateral gland involvement, unilateral gland gradually expanding to bilateral glandular lobes, and local single lobe involvement [11]. The clinical misdiagnosis rate of thyroid diseases is as high as $7.15 \%$; especially, the differentiation of hyperthyroidism, subacute thyroiditis, and lymphocytic thyroiditis is very difficult [12]. A SPECT scan of the thyroid provides a complete picture of the thyroid's position, size, shape, and radioactivity. The abnormal changes of thyroid morphology can be observed intuitively, which is applied in clinical practice for the diagnosis of thyroid diseases [13]. 




FIGURE 8: ROC curve of SPECT for diagnosing thyroid diseases.

${ }^{99} \mathrm{TcO}_{4}$ and ${ }^{131} \mathrm{I}$ have similar chemical properties and can be absorbed by thyroid tissue. The reason for the adoption of ${ }^{99 \mathrm{~m}_{\mathrm{TcO}}}$ as the imaging agent was its short halflife, which is about $6.21 \mathrm{~h}$, and the radiation energy is about $140 \mathrm{keV}$. Moreover, ${ }^{99 \mathrm{~m}} \mathrm{TcO}_{4}$ itself does not participate in the synthesis of thyroid hormones, which can objectively reflect the uptake capacity of the thyroid, and is suitable as a SPECT imaging agent [14]. ${ }^{99 \mathrm{~m}} \mathrm{TcO}_{4}$-SPECT imaging analysis was conducted on 35 patients with thyroid disease. The results showed that 7 cases $(20 \%)$ of thyroid pathology showed local lesions, of which 2 cases had no imaging (5.71\%) and 5 cases had sparse imaging (14.29\%). There were 12 cases (34.28\%) of single leaf lesions, of which 3 cases (8.57\%) were without imaging and 9 cases $(25.71 \%)$ were sparsely imaging. There were 16 cases (45.71\%) of double leaf lesions, of which 6 cases (17.14\%) had no imaging and 10 cases $(28.57 \%)$ had sparse imaging. Thyroid tissue was not visible or sparsely visible, and the boundaries of the glands were not clear. The complete thyroid morphology cannot be displayed. The morphology was not regular, and the radioactive uptake was uneven, which suggested that the degree and scope of abnormal thyroid changes can directly affect SPECT imaging [15-17].

The VITU of the region of interest between the thyroid gland and the soft tissue around the neck, the submandibular gland, and the parotid gland in the observation group and the control group were calculated. The results showed that the VITU of thyroid/neck in the observation group $(2.68 \pm 1.32)$ was remarkably inferior to that in the control group (12.347.54). The VITU of the thyroid/submandibular gland in the observation group $(1.02 \pm 0.41)$ was remarkably inferior to that in the control group $(8.89 \pm 4.86)$. The VITU of the thyroid/parotid gland in the observation group $(1.04 \pm 0.58)$ was remarkably inferior to that in the control group $(8.53 \pm 4.25)$. The differences were all statistically considerable (all $P<0.05$ ). It was suggested that, in the early stages of thyroid disease, thyroid follicles were destroyed and the glands' ability to absorb technetium was greatly reduced. In contrast, the thyroid's surrounding soft tissues, including the mouth, neck, submandibular, and parotid glands, had a relatively high uptake of technetium. Therefore, the calculated visual index of the thyroid gland showed a considerable decrease [18]. The VITU in this study was based on the VITU of the thyroid/submandibular gland. The simple operation of mapping the region of interest of the submandibular gland can successfully avoid the effects of oral radiation, which was consistent with the results of Razavi et al. [19].

Currently, SPECT imaging is widely utilized in bone, neural network, cardiovascular, and endocrine fields. Benign and malignant tumors' accurate positioning, differentiation, evaluation, and evaluation of the efficacy of the evaluation can provide a reference for clinical treatment plan. In this study, the sensitivity, specificity, accuracy, positive predictive rate, and negative predictive rate of ${ }^{99} \mathrm{TcO}_{4}$-SPECT in the diagnosis of thyroid disease were $95.2 \%, 90.3 \%, 91.5 \%$, $94.3 \%$, and $89.6 \%$, respectively. The area under the curve of ${ }^{99} \mathrm{mcO}_{4}$-SPECT for thyroid disease diagnosis was 0.958 , with a $95 \%$ confidence interval of 0.834 to 1 , which indicated that SPECT imaging can accurately locate the lesion and obtain anatomical information, greatly reducing the misdiagnosis rate of ${ }^{99} \mathrm{TcO}_{4}$ imaging, which was consistent with the results of Dong et al. [20].

\section{Conclusion}

The results showed that the $\mathrm{CNN}$ algorithm proposed in this study had a good segmentation effect. SPECT imaging can accurately locate the anatomical information of thyroid disease and have high sensitivity and accuracy for the lesion site. However, the weakness of this study lies in the small sample size, which may lead to selection bias. Therefore, it is necessary to improve and expand the sample size for further analysis and research in the later stage. In conclusion, the segmentation effect of SPECT image based on the CNN algorithm in this study is good, which provides a reliable reference for clinicians to improve the treatment plan of thyroid disease.

\section{Data Availability}

The data used to support the findings of this study are available from the corresponding author upon request.

\section{Conflicts of Interest}

The authors declare no conflicts of interest.

\section{References}

[1] W. W. Lee and K.-S. Group, "Clinical applications of technetium-99m quantitative single-photon emission computed tomography/computed tomography," Nuclear Medicine and Molecular Imaging, vol. 53, no. 3, pp. 172-181, 2019.

[2] J.-Y. Kim, J. H. Kim, J. H. Moon et al., "Utility of quantitative parameters from single-photon emission computed tomography/computed tomography in patients with destructive 
thyroiditis," Korean Journal of Radiology, vol. 19, no. 3, pp. 470-480, 2018.

[3] J. Kim, H. Lee, H. Lee et al., "Quantitative single-photon emission computed tomography/computed tomography for evaluation of salivary gland dysfunction in s syndrome patients," Nuclear Medicine and Molecular Imaging, vol. 52, no. 5, pp. 368-376, 2018.

[4] Z. Chen, J. Fu, Q. Shao, B. Zhou, and F. Wang, "99mTc-MIBI single photon emission computed tomography/computed tomography for the incidental detection of rare parathyroid carcinoma," Medicine, vol. 97, no. 40, Article ID e12578, 2018.

[5] K. Agrawal, P. Sradha Patro, and K. Behera, "Graves' disease in eutopic thyroid with ectopic mediastinal thyroid tissue: role of single photon emission computed tomography-computed tomography," Indian Journal of Nuclear Medicine, vol. 34, no. 3, pp. 230-232, 2019.

[6] J. Y. Lee, H.-S. Song, and M. Kang, "Physiological uptake of radioactive iodine around an artificial eyeball observed with single-photon emission computed tomography/computed tomography after radioactive iodine treatment," Nuclear Medicine and Molecular Imaging, vol. 54, no. 4, pp. 204-206, 2020.

[7] H. Y. Suh, H. Y. Na, S. Y. Park et al., "The usefulness of maximum standardized uptake value at the delayed phase of Tc-99m sestamibi single-photon emission computed tomography/computed tomography for identification of parathyroid adenoma and hyperplasia," Medicine, vol. 99, no. 28, Article ID e21176, 2020.

[8] J. Lee, H.-S. Song, J. Choi et al., "Detecting synchronous parathyroid adenoma and false-positive findings on technetium-99m MIBI single photon-emission computed tomography/computed tomography," Diagnostics, vol. 9, no. 2, p. 57, 2019.

[9] L. Ma, C. Ma, Y. Liu, and X. Wang, "Thyroid diagnosis from SPECT images using convolutional neural network with optimization," Computational Intelligence and Neuroscience, vol. 2019, pp. 1-11, Article ID 6212759, 2019.

[10] E. K. Alexander, E. N. Pearce, G. A. Brent et al., "2017 guidelines of the American thyroid association for the diagnosis and management of thyroid disease during pp," Thyroid, vol. 27, no. 3, pp. 315-389, 2017.

[11] Q. Liu, H. Pang, Q. Liu, J. Zhou, and Y. Liu, "Preparation of human single-chain variable fragment antibodies against anaplastic thyroid carcinoma, and single photon emissioncomputed tomography/computed tomography imaging in tumor-bearing nude mice," Oncology Reports, vol. 37, no. 5, pp. 2980-2986, 2017.

[12] D. Çayir, M. Araz, A. Yalçindağ, and E. Çakal, "The relationship between semiquantitative parameters derived from technetium-99m metoxyisobutylisonitrile dual-phase parathyroid single-photon emission computed tomography images and disease severity in primary hyperparathyroidism," Nuclear Medicine Communications, vol. 39, no. 4, pp. 304311, 2018.

[13] R. Lee, Y. So, Y. S. Song, and W. W. Lee, "Evaluation of hot nodules of thyroid gland using Tc-99m pertechnetate: a novel approach using quantitative single-photon emission computed tomography/computed tomography," Nuclear Medicine and Molecular Imaging, vol. 52, no. 6, pp. 468-472, 2018.

[14] M. R. Kim, S. Jo, and H.-k. Shim, "Port-site implantation diagnosed by iodine-131 post-ablation single-photon emission tomography-computed tomography after robotic thyroidectomy: a case report," American Journal of Case Reports, vol. 20, pp. 1695-1698, 2019.
[15] C. Zwarthoed, K. Chatti, J. Guglielmi et al., "Single-photon emission computed tomography for preclinical assessment of thyroid radioiodide uptake following various combinations of preparative measures," Thyroid, vol. 26, no. 11, pp. 1614-1622, 2016.

[16] M. Araz, D. Çayir, M. Erdoğan, B. Uçan, and E. Çakal, "Factors affecting the sensitivity of Tc-99m methoxyisobutylisonitrile dual-phase parathyroid single photon emission computed tomography in primary hyperparathyroidism," Nuclear Medicine Communications, vol. 38, no. 2, pp. 117-123, 2017.

[17] H. Kasahara, M. Sato, S. Nagamine, K. Makioka, K. Tanaka, and Y. Ikeda, "Temporal changes on 123I-i and cerebral blood flow single-photon emission computed tomography in a patient with anti-N-methyl-D-aspartate receptor encephalitis," Internal Medicine, vol. 58, no. 10, pp. 1501-1505, 2019.

[18] L. Jiang, Y. Xiang, R. Huang, R. Tian, and B. Liu, "Clinical applications of single-photon emission computed tomography/computed tomography in post-ablation 131iodine scintigraphy in children and young adults with differentiated thyroid carcinoma," Pediatric Radiology, vol. 51, no. 9, pp. 1724-1731, 2021.

[19] S. Razavi, B. Ziebarth, R. Klein, and W. Zeng, "Dual timepoint quantitative SPECT-CT parathyroid imaging using a single computed tomography," Nuclear Medicine Communications, vol. 39, no. 1, pp. 3-9, 2018.

[20] F. Dong, L. Li, Y. Bian et al., "Standardized uptake value using thyroid quantitative SPECT/CT for the diagnosis and evaluation of graves' disease: a prospective multicenter study," BioMed Research International, vol. 2019, Article ID 7589853, 8 pages, 2019. 